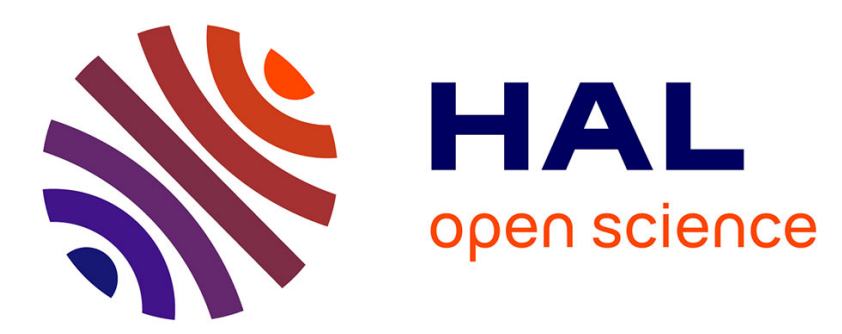

\title{
La politique de régionalisation et de libéralisation ferroviaire en Allemagne, l'exemple de la Basse-Saxe Jean-Pierre Wolff
}

\section{To cite this version:}

Jean-Pierre Wolff. La politique de régionalisation et de libéralisation ferroviaire en Allemagne, l'exemple de la Basse-Saxe. Annales de géographie, 2005, 646 (2005/6), p. 602-616: bibliogr., carte. hal-01814145

\section{HAL Id: hal-01814145 \\ https://hal-univ-tlse2.archives-ouvertes.fr/hal-01814145}

Submitted on 12 Jun 2018

HAL is a multi-disciplinary open access archive for the deposit and dissemination of scientific research documents, whether they are published or not. The documents may come from teaching and research institutions in France or abroad, or from public or private research centers.
L'archive ouverte pluridisciplinaire HAL, est destinée au dépôt et à la diffusion de documents scientifiques de niveau recherche, publiés ou non, émanant des établissements d'enseignement et de recherche français ou étrangers, des laboratoires publics ou privés. 


\title{
La politique de régionalisation et de libéralisation ferroviaire en Allemagne, l'exemple de la Basse-saxe
}

\author{
The politics of regionalization and liberalization \\ of railway in Germany, the Niedersaxen case
}

Jean-Pierre Wolff

Prolesseur de gépgraphie ot aménagement.

Universite de Toulouse le Mirail, CIRUS-CIEU

Résumé La libéralisation des transports publics, dans le cadre de la dérégulation prônée par Bruxelles conceme tous les pays européens selon des modalités qui leur sont propres. En Allemagne, ce processus se met en place sous une double conjoncture tant économique qu'environnementale. En effet, le souci de libéraliser ce secteur accompagne une forte demande sociale en matière d'écologie, d'ailleurs les Vells entrent au gouvemenent fédéral en 1998. Ces deux aspirations se conjuguent à um moment sensible lié à la réorganisation des transports ferroviaires de voyageurs en particulier sur les plans locaux et régionaux.

Abstract Within the liamework of the deregulation lauded by Brussels, the liberalization of public transportation concerns all European countries, but can be implennented as each country sees it fit. In Germany, this process is set up rollowing a double intake: economic and environmental. Indeed, the liberalization dive in this sector comes along with a strong social dennand in ecology (the Greens entered the rederal govemment since 1998). These wo aspirations conjugate at the sensitive moment when the traveler railway system was being reorganized, at the local and regional level in particular.

Mots-clés Allemagne, Basse-Saxe, transports ferroviaires de proxinnité, régionalisation, libéralisation.

Key-words Gemamy, Niedersachsen, milway transponation of proximity, regionalization, liberalization.

En Altemagne, les aurorités territoriales exercèrent jusquà une période très récente, l'ensemble des services de transport public. L'Etat fédéral assurait par l'entremise de la Bundesbahn - BB - la gestion quasi rotale du réseau ferroviaire. Les villes possèdent encore à ce jour leur compagnie de transport en commun, financéc par le budget local et recueillent les profits réalisés de la commercialisation de services - eau, electricité, gaz... gérée par les services industriels communaux. Lat libéralisation menace ce dispositif ex remet en cause le service public de transport en commun. Sur le plan feroviare, les mutations sont nettes. La privatisation des chemins 
de fer allemands, par la création d'une société anonyme dont l'État possède jusqu'à présent tout le capital, fut facilitée par la fusion des deux réscaux ferroviaires différents et complémentaires, lors de la réunification de l'Allemagne en 1989 et la création en 1994 de la Deutsche Bahn AG - DB AG - et elle va de pair nvec l'introduction de la régionalisation des transports de voyageurs. Ceci se traduit par des changements importants en particulier au niveau des lignes locales et régionales, dont certaines ont été fermées on reprises pax des länder, des communes et des sociétés privées. Cette érolution importante remet en jeu les politiques territoriales traditionnelles dans lesquelles les rôles des acteurs sont redéfinis avec en particulier l'entréc de prestataires privés. En même temps clle s’inscrit dans un pragmatisme qui caracterise les décisions en matière d'aménagement du territoire et de développenent local et régiomal. En cffet, ce partenariat publicprivé peut se conceroir comme une réponse à une situation rigide et dépassée en termes d'organisation et de gestion des dessertes ferroviaires locales et régionales, d’articulation entre des politiques de déplacement des régions plus ou moins urbanisées et celles des agglomérations proches et enfin comme un moyen d'introdure une plus grande réactivité aux politiques de transports et de développement territorial.

\section{La régionalisation des transports de proximité}

Les réformes structurelles, caractéristiques de la fin du xye et du début du $x_{1}$ siècle ont de nombreuses incidences en termes économique, social, idéologique ef territorial. Pour illustrer ce propos, prenons l'exemple de la déréglementation des transports et plus précisénent des services ferroviaires locaux. Sans rappeler en amont les philosophies en jeu, il est important d'analyser un exemple choisi et localisé en Basse-Saxe pour comprendre les enjeux territoriaux qui en découlent. Cependant avant d'aborder ces points rappelons la mise en place de cette réforme capitale.

L application des directives européennes en matière de libéralisation des transports collectifs passe par un changement conséquent dans l'organisation des dessertes ferroviares qui ne dépendent plus de la DB pour le trafic local et régional mais de l'autorité des Länder, seule reconnue. L'entrée en vigueur de la loi rérganisant les chemins de fer, Gevtz zup Nounhmung des Eisenbahnuesens du 27.12.1993 constitue le premier pan d'une renise à plat des politiques de déplacement et en particulier dans le domaine ferroviaire. Ce premier pas fianchi, il est rapidement complété par la Regioualisionng des affentieluen Personennabuetkehs du 01.01.1996, contenant en particulier des articles relarifs à la régionalisation du trafic ferroviare de proximité. Cette evolution croise régionalisation, obligation de dessertes locales et régionales, affumation d'un mouvemen écologiste structuré et libéralisation. Ia régionalisation de certains services ferroviares offre des possibilités de gestion plus souple du rafic local et incite les collectivités et les entreprises privées à ajuster len service aux besoins des populations 
concencécs. La régionalisation se traduit par une forte croissance de lofle en kn/rain, qui passe de 498 à 570 millions entre $1993-1994$ et 1999 2000. Celle-ci vise à décharger la DB de son obligation à poursuive son activité ferroviare sur des lignes en général déficitaires. I_es appels d'offre lancés par les autorités régionales on locales pour reprendre les semices de la DB ont permis à celle-ci de récupére $50 \%$ des relarions soumises à cette procédure, les aurres $50 \%$ revenant à des entreprises privées, ì des sociétés publiques régionales ou à des sociétés d'économie mixte. Le fait notable est lintroduction importante de sociétés privées dans ce crencau des transports terroviaires locaux. Limportance des unapports publics dans les migrations pendulaires - 50\% des parts de ce marché -, explique cet engouement pour le ransport ferroviare, seul à même de décongestionner cerraines grandes métropoles et participer à la lute contre la pollution atmosphérique. Cependant cette présence de groupes privés puissants et en particulier émangers, poumait laisser craindre, comme ce fut le cas au Royaume-Uni, soit un démantèlement total de la Db, soit une politique de dumping dans les années qui viennemi et qui se manifesterait dans les deux hypothèses par une baisse de la qualité des prestations ferrovianes. Nous verons que ces craintes ne sont pas pereeptibles dans cette ouverture à la concurence ferroviaire sur le plau régional.

Avec la réforme de 1996 , le Bund verse directement aux Länder 7 milliards d'euros par an pour couvrir les charges d'exploitation des lignes locales. Cetre somme augmentée de $1,5 \%$ par an se décompose en 3,6 milliards qui comvent les deficits dexploitation et en 3,4 milliards d'euros qui servent à soutenir la modernisation des réseanx locaux. Contranement aux Régions en France, les Iänder peuvent choisir une ou plusieurs autorités organisatrices des tansports; de ce fat est introduite une certaine antonomie par rapport au Land. Ce n'est plus ke Land qui est directement responsable des services ferroviates régionaux mais cette ou ces autorité(s) organisatrice(s). Lautorité organisatrice dispose d'une grande souplesse ef d'une grande réactivité par rapport aux évolutions des tertitomes placés sous son autorité. Les périnzères géographiques de ces autorités organisatrices comespondent anx grands bassins de vie et tiement compre des disparités entre espaces fortenent et peu urbanisés pour corriger les dysfonctionnements liés à des siruarions de développement trop diferentes. Enfin les Länder au nom de leur automonic et des policiques publiques empreintes de pragnatisme, peuvent conduire des démarches uès différentes les unes des autres, c'est ainsi que certains Länder souhaitent gérer directement leur tramsport terroviaire, d'autres délèguent après lancement d'appel d'offres leur service au mieux disant.

Pour illustrer cette organisation prenons les cas suivants. Le Land Rhénanie du Nord Wesphalic compte neut autoriés organisaurices liées à des découpages fonctionnels, le Land Rhénanie-Palatinat senlement deux et le Land Basse-Saxe trois autorités orgmisatrices. Dans ce dermier exemple, lautorité organisatrice au périmètre le plus raste couve envion les $80 \%$ 


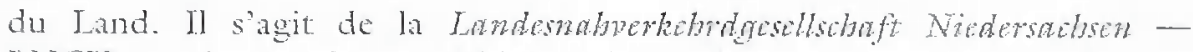
ILNGV - mise en place en 1996, à côté de laquelle deux autres sont centrées sur les grandes agglomérations urbanes aurour d'Hanove avec le Krex Gros Hamoner - KGI- - et le Zwecherbnud Grosmom Bramschmeig - ZGB - pour la région multipolaire de lirunswick, Wolfsburg ex Salzgitter. Cette politique régionale est appliquée au plus près du verrain en prenant en considération l'ensemble des disparités de peuplement, de développement socio-économique ét des besoins différenciés de mobilité. Les antorités organisatrices nettent en place concrètement les grandes orientations des politiques de déplacement des Länder. Elles sont chargées de deux domaines. Le premier, porte sur les politiques glohales de déplacement avec la définition en termes de choix d'investissement pour la conforter et la rendre plus dyminique dans le cadre des directives de Bruxelles et en particulier elles sont responsables de définir les appels doffres et: d'instruire les réponses. Ie sceond concerne l'aspect financier de cette politique qui est déterminant pour l'efficacié et le renforcement des transports locaux. Enfin ces autorités organisatuices détiennent un pouvoir décisionnel considerable en termes de choix d'opérateurs, de matériels et d'interventions sur l'infrastructure. Ce modèle allemand diffère à la fois de ce qui se passe en France et au Royume-Uni. Il est important de souligner la responsabilité des pouvoirs régionaux à travers les autorités organisatrices chargées de détinir une politique publique des transports de proximité, même si celle-ci est conduite par des actems différents, publics ou privés, voire quelquefois les associant dans des opérations de partenariat privépublic. Ce souci d'accorter par le Land unc autonomie aux autorités organisarrices correspond à un double souhait. Le premier repose sur le choix d'une politique territoriale d'anénagenent décentralisée qui délègue aux niveaux les plus pertinents les compétences appropriées. Cette démarche est étroitement intégrée à la politique globale du liand et de ce fait elle šarticule avec celles concemant les autres espaces de ce hand. Le second, découlant du premier, renforce lin responsabilisation des autorirés organisatrices en termes de recherche consensuelle entre les diflérents acteurs politiques, économiques et associatifs en s'inscribant dans un processus de gestion territoriale très fine pour tenir compte des spécificités locales en matière de localisation des populations, des activités et des politiques de déplacement, le tout dans un cadre ou une injonction de déseloppement durable.

L'entrée en vigueur de la régionalisation place théoriquement sur le même pied les opérateurs privés et publics qui répondent à des cahiers des clarges précis élaborés par chaque Land. Done l'organisation des transports ferroviares varie d'un Land à l'autre, même si elle s'inscrit dans les grands principes favorisant lutilisation optimale des modes collectifs. Cependant certains Iänder contrôlent étroitemen leur polinique par le recours à la DB comme opérateur ou à des sociétés locales ou régionales, d'autres préfèrent déléguer ces services à des collectivités territoriales plus ou noins importantes comme à Hanove ou à Stuttgart et enfin certains Länder recouren 
aux services de sociétés privées allemandes ou étrangères conforménent aux décisions européennes en la matière. Rappelons que des sociétés privées de perite taille ont toujours été présentes face à la DB. Il s’agissait essentiellement de réseaux municipaux ou industriels complétant celui de la DB. Mais très récemment d'autres compagnies privées ou mixtes sont nés pour reprendre dans le cadre de la loi de 1996. lexploitation de plusieurs lignes ferroviaires secondaires. C"est à travers l'une des plus ancicnnes er importantes compagnies ferroviaires privées allemandes, la Deutsche Eisenbahngesellschaft - DEG - rachetée par une filiale de la Conpagnie Générale des Eaux, société francaise devenue Vivendi puis Veoha er dont le secteur des transports porte le nom de CONNEX depuis quelques années, que ce processus de privatisation comnait un cssor rapide. Ce groupe multinational contrôle plusicurs perits réseaux locaux en Allemagne et cst devenu le premier operateur: privé du pays et le second globalement, certes encore tres loin derrière la DB. Début 2004, il gère une quarantaine d'entreprises de transport appartenant aussi bien au domance ferrovare qu'à celui des réscaux de uansports urbains et emploie environ 3330 personnes en Allemagne.

\section{L'expérimentation en Basse-Saxe}

Pour illustrer ce processus, nous prenons un exemple des plus instructifs concemant la mise en place de la pemière privatisation dans le Land de Basse-Saxe 1. Rappelons cependant qu'il ne s'agit pas de la prenière privatisation en Allemagne. Au début des annés 1990 avant l'enuée en vigueur de la loi de 1996, une société privée, la Eisenbahnen und Verkehrsherricbe Elhe-Weser (EVB) a réexploité la ligne Hambourg-Buxtehude-Bremerhaven que la DB voulait fermer. Dans le cas de la Basse-Saxe, analysons comment: se réalisent ces opérations complexes, qui dans notre exemple débouchent sur une véritale opération de partenariat public-privé, dans laquelle les deux parties ont tout à gagner. Quels en sont les acteuss et quelles stratégies emploient-ils pour arriver ì ce but?

le Land de Basse-Saxe, le plus vaste d'Allemagne après la Bavière avec $47600 \mathrm{~km}^{2}$ compte une population denviron 7,8 millions d'habitants $\mathrm{et}$ une densité de 1.66 habitants au $\mathrm{km}^{2}$ inférieure i celle de l'Allemagne (231 ha/ $\left./ \mathrm{km}^{2}\right)$. Dans ce contexte, la politique daménagement du territoire jour un róle très important. Elle sappuie sur un concept d'ensemble qui repose sur la hiérarchisation des activités et la planification basce sur ha concentration décentralisée.

1 Cel article reprend une communication intilulee La politique de privalisation femoviame en Basse-Saxe, non publiée hate à Dijon dans le cadre du Colloque "Les effets spaliaux el territoriaux de la "dêegelententation" des transports en France en Europes. les 14 et 15 septembre 2000. Une nise à jour remontant à decembre 2004 a été laite el elle s'appuie parliellenent sur un vovage d"útude, une mise à jou bibliographique el le suivi d"um travail de recherche intitula La polinque de liberalisation ferroviaire en Allemagne: lexemple de la Norówestbahn en Basse Saxe, effectue sous ma responsabilité par Sylvain Séguret au Département de géographie de l'université d'Orléans at soutenu en octobre 2003. 


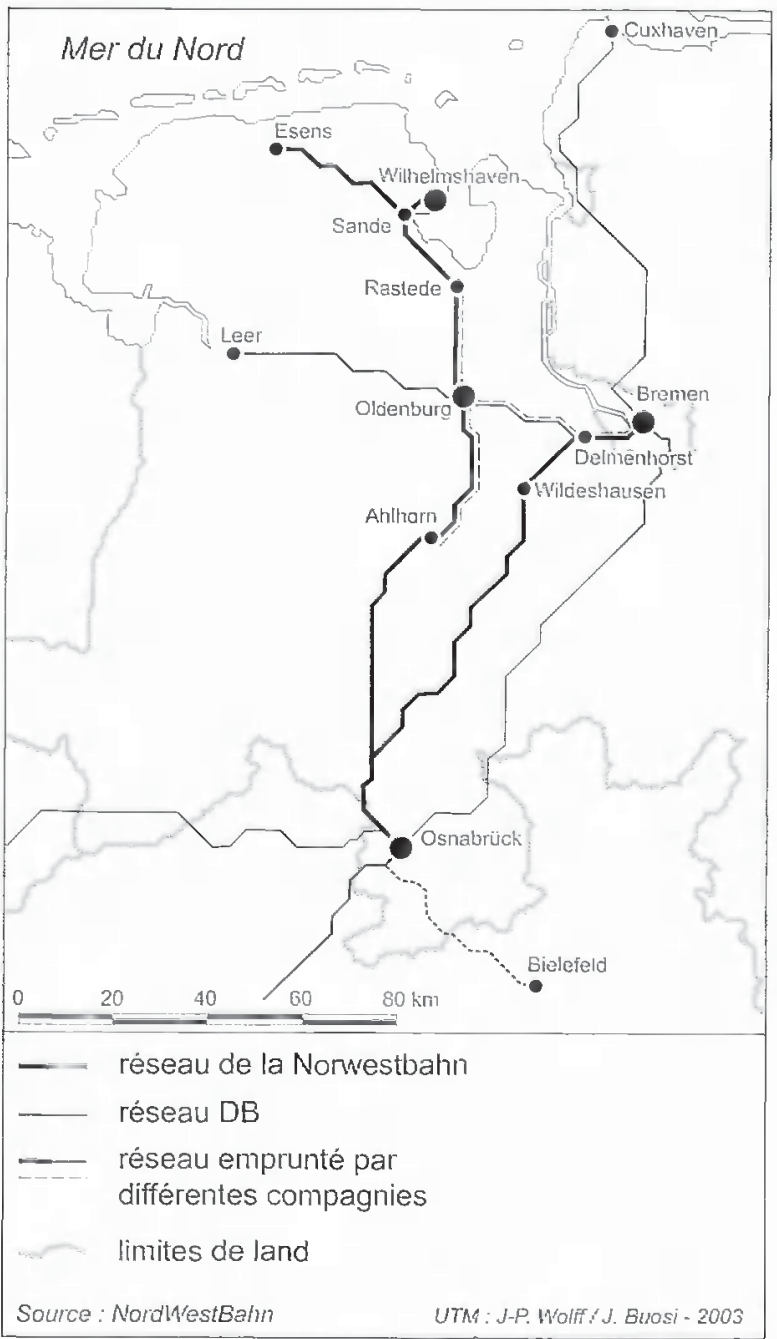

Fig. 1 Le réseau initial de la NWB en 2000.

The initial newrork of the NMB in 2000.

Cette approche très fonctionnelle et pragmatique de laménagement du territoire et qui sinscrit dans une longue tradition de hiérarchisation des espaces et des activités implique une gestion très fine de l'ensemble des déplacements pour optimiser le développement économique tout en prenant: en compte les contraintes budgétaires et écologiques de plus en plus sévères. En 1996, le gonvernement de Basse-Saxe a crée, pour faciliter la modernisation du trafie régional, les trois autorités oranisatrices des trans-

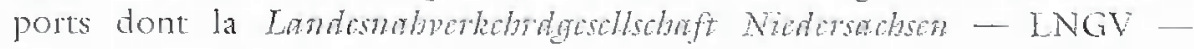


l'organisme des transports de proximité du Land, qui intervient en amont dans les politiques de déplacement. Le land a mis a sa disposition un budger important pour mener à bien cette politique entre 1998 at 2001. Environ 71,4 millions de DM servent à l'amélioration des gares et des arrêts, 118 mitlions de DM atribues aux infiastructures ferrovianes permetent des diminutions des temps de parcours et 78,5 millions de DM sont consacrés à lacquisition de matériels neufs.

Pour la première expérience dans ce Land, Ia l NGV a voulu montrer que l'on pouvait reprendre des lignes jugées sans avenir par la 10l3. Pour cela il a été proposé un regroupement de rois lignes aux uafics bien différents atin de compenser la taiblesse de certaines sections par la fréquentation plus importante d'autres, dans un Trilnets - parties de réseau centré sur Osmabrück. Ce réseau (fig. 1.) se localisait au départ dans un secteur géographique de $7630 \mathrm{~km}^{2}$ et de 1300000 habitants compris entre la mer du Nord ex Osnabrück rouchant rois lignes ferroviaires exploitées par la Dl3:

- Osnabrück-Oldenburg-Wilhemshaven, longue de $165 \mathrm{~km}$;

- Wilhemshaven-Sande-Esens, longue de $41 \mathrm{~km}$;

- Osnabrïk-Delmenhorst-Bremen, longne de $112 \mathrm{~km}$.

En mars 1997, dans un premicr temps, le lancement d'un appel d'offre initié par la LNGV de Basse-Saxe intéresse six sociétés, parmi lesquelles la DB, la DEG associée à la Stahturerk Osnabrïle - SWO - Scrvices

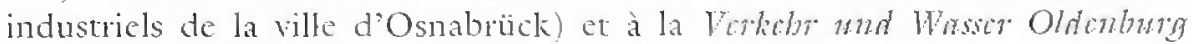
- VWG - (Senvice des ransports publics et de l'eau de la ville d'Oldenburg), la Compagnie de transport de brểne et trois autres sociétés mineures. Dans une seconde phase, ces trois dernières se retirent et il ne reste en lice que les rois premières. Puis la DB et la Compagnie de transport de Brềme proposent un projet commun d'exploitation face à celui de la DEG, de la SWO et de la VWG. En détinitive c'est la proposition de ces trois dernières qui est retenue. À partir de là, es trois partenaites créent la NordWostBabn - NWB - chargéce d'exploiter le réseau concédé.

En mai 1998 la LNGV accorde l'exploitation des trois lignes dans la région Ems-Weser, soit $318 \mathrm{~km}$, ì la NWB, dont le siège et les atelier's se tronvent à Osnabrück. Son capinal se répartit entre la DEG (64\%), la SWO (26\%) et la VWG (10\%). La NWB est une filiale de la Dentsorr Eiscubabngesellschaft. C'est une nouvelle entité juridique privée regroupant deux partenaires publics êt une société privéc appartenant à Vivendi qui possédait déjà, à cette époque, 16 compagnies ferroviaires dans d'atutes Länder. Son expérience dans ce domaine représente un atout non négligeable pour l'attribution d'une concession. Ia NWI3 avec seulement 70 employés dont 50 conducteurs, commence le 5 novembre 2000 l'exploitation de ce nouveau semice ferroviaire de proximité.

la régionalisation et la libéralisation de l'offre ferroviaire, conduites de concert ont rencontré de nombreuses difficutés institutionnelles et organi- 
sationnelles. Dans le cas étudié, la complexité juridique du processus découle d'une multitude de partenaires qui exercent une autolité sur la mise en place des services ferroviaires. Plusieurs länder sont concenés et leurs préoceupations en matière de transport de proximité peuvent êre différentes mêne si leur philosophie ess identique. Par exemple au nord d'Osnabrück, sur le trone commun des deux lignes, sept kilomètres sont situés dans le Land Nordrhein-Westfalen et à l'extrémité de la relation Osnabrück-Dehmenhorst-Bremen, les demiers kilomètres se trouvent dans le Land de Brếme. Chaque Land délègue la gestion de ce rype de rrafic à un organisme qui lui est propre, mais à côté des services administratifs régionaux, d'autres exercent des compétences dans le mème secteur, comme les structures communales ou supra communales de transpon de voyageurs. L'ensemble de ces intervenants différents composent avec la DB, qui elle-même est scindée en plusicurs entités propres comme la DB lmmobiliert pour les gares, la DB Rate mat Tombtik pour la commercialisation des différents produits ou le secteur infrastructure de la DB. Le tout est regroupé dans un holding. Ia NWB s'acquite auprès de la DB d'un péage pour l'utilisation des voies er des points d'arrêt.

Pour être un peu plus concret quant aux difficultés rencontrées, prenons le tronşon final de la ligne Osnabrück-Delmenhorst-13remen, oì circule entre ces detux dernières villes des comvois de la DB, de la Compagnie de transport de Breme et de la NWB. Entre Rastede et Alhorn sur la relation Osnabrück-Oldenburg-Wilhemshaven se croisent les trains des rois transporteurs mentionnés plus haut er entre Oldemburg er Alhorn seulement ceux de la Companic de transport de Brêne et de la NMB. Certe juxtaposition de sociétés différentes sur des infrastructures qui appartiennent encore toutes a la DB, impose une multiplication des accords et des conventions pour sumonter de nombreux motifs de friction face à la toute puissance de la D13. La NW13 doit suive un cahier des charges extrêmement précis imposé par la LNGV. Elle ne possède ni les roies et les báriments relevant de la DB ni les trains qu'elle loue au land de Basse-Saxe. Dans l'avenir la NWB souhaiterait pouvoir acquérir l'ensemble des infrastructures ou plus exactement la rétrocession de celles-ci à la LNGY qui autoriserait une plus grande souplesse d'utilisation en temes de péage et d'offre supplémentaire de sillons de circulation pour ses urans. Lachat des gares par le Land permettrait leur modernisation parallèlement à celle réalisée par l'achat d'un pare d'autorails neufs et le renouvellement particl de la voie.

\section{La nouvelle offre ferroviaire de la NWB}

L'appel d'offe remporté par la NWB se traduit par une convention particulièrement précise ct exigeante. Le Land à uavers la l NGV contrôle très étroitement cetre première expérience, qui stipule les points suivants: 
- le land définit les horaires;

- le Land rétribue la NWB pour le service rendu;

- Le réseau est entretenu par la DB;

- la NWB s'acquitte d'un droit pour l'utilisation des infrastructures de la DB;

- Je matériel appartient au Land;

- la NWB loue ce matériel auprès du Land;

- ees nouvelles prestations se font sur le coutt de celles de la Dl3 du service d'hiver 1997-1998;

- la convention initiale est valable cing ans et ensuite elle est renégociable pour une période de 1.5 ans.

La NWb a pu s'imposer par une offe ferroviaire basée sur l'augmentation des circulations, une baisse des remps de parcours, l'introduction du cadencement et le tout à un coût identique pour le land par rapport aux prestations de la Dl3 en 1997-1998. Globalement le service de la NWB apporte une plus-value au Land par rapport à la situation précédente.

Les dessertes régionales visent une réduction de la durée du voyage. Sur la relation Osnabritck-Oldenburg-Wilhemshaven (165 km), et comptant 17 arrêts intermédiaires, le temps de parcours diminue de 35 minutes, passant de deux heures cinquante minutes à deux heures quinze. Sur celle de Osnabrück-Delmenhorst-Bremen (11.2 km), le gain de temps est de 25 minutes et enfin sur la dernière Wilhennshaven-Sande-Esens $(41 \mathrm{~km})$ celui-ci est de 15 minutes. La relation directe Osnabrück-Sande-Esens qui ne dessert pas Wilhemshaven est raccourcie d'une heure. Cette amélioration importante des temps de parcours dépend avant tout de la modernisation de l'infrastructure, d'une gestion optimale des sillons et de la mise à disposition d'un nouveau natériel. Le cadencement et l'amplitude des services renforcent lefficacité de la NWB et répondent à une demande de la clientèle. Complétant cet aspect, la LNGV a décidé d'améliorer les infrastructures qui appartiennent à la $\mathrm{DB}$, en particulier pour relever les vitesses commerciales, accroitre le confort des passagers et fluidifier le trafie ferroviaire. La reconstruction de la voie à la charge de la LNGV entre Osnabrïck et Oldemburg permet d'augmenter la vitesse de 80 à $120 \mathrm{~km} / \mathrm{heure,} \mathrm{car} \mathrm{sur}$ certaines sections par manque de soin de la DB, la vitesse autorisée était limitée à $50 \mathrm{~km} /$ heure. L'organisation des sillons augmente les possibilités de circulation du nombre de convois en particulier sur ces Jignes à roie unique.

La convention entre la NWB et la LNGV impose une forte amélioration des services sur les différentes lignes exploitées par la NWB en prenant comme base de référence le service d'hiver 1997-1998 de la Dls avec 2,4 millions de kilomètres/train, qui passe à 3,1 millions de kilomètres/ train au service 2000-2001 pour un réseau de 318 kilomètres comportant quarante arrêts. En 2002, la NWB assume 3,5 millions de train/km et transporte 4,5 millions de passagers contre 2,6 millions en 1998 par la Dl3. Le service cadencé est introduit sur l'ensemble des lignes avee un train par 
heure sur les relations Osnabrück-Delmenhorst-Bremen et OsnabrückOldenburg-Wihemshaven et entin une dessente aux deux heures, et à l'heure en période de pointe, pour celle de Wilhennshaven-Sande-Esens. Les possibilités de correspordance sont renforcées avec tous les lnterregio de la $D B$ à Osnabrück et à Oldenburg. Un an après l'inauguration du nouveau réscau, les premiers résultats montent l'effrcacité de cette politique qui a ₹u la fréquentation bondir de $70 \%$ dépassant de loin les estimations les plus optimistes de la NWB.

\section{Le renforcement de cette politique ferroviaire}

Devant ce succès inespéré, la LNGV renouvelle l'expérience toujours à partir du même processus d'appel d'offres er du même cahier des charges pour la convention du service ferroviaire. D'autres Teilmetz (fig. 2.) sont constitués par la LNGV et les autorités organisatrices des liander de brême et de Hambourg pour la mise sur pied d'un service recoupant les périmères d'intervention des trois autorités organisatrices. Il s'agit des lignes suivantes:

- Uelzen-Hambourg-Brềne;

- Münster-Bielefeld-laderborn-Holzminden;

- Bodenburg-Hildesheim-Hameln-Löhne;

- Bielefeld-Osnabrück.

Le réseau Uelzen-Hambourg-brène est long de 197 kilomètres et concerne trois Länder et trois autorités organisatrices différentes, la LNGV couve 170 kilomètres de ce nouveau réseau. Ises autorités organisatrices ont retenu comme exploitant un groupement de compagnies régionales sous la houlette de la Osthannoverschen Eisenbahn - OHE - qui a commené son service commerial en 2003 et qui partage avec les trains de la $\mathrm{DB}$, les infrastuctures de cette demière. Ce service correspond à un express régional qui ne dessert que quelques gares.

Ice Tcilncto Weserbahn-Lammetalbahn reliant les villes de Bodenburg, Hildeshein, Hamehn et Löhne se développe sur 119 kilomètres. Les deux mênes Länder sont concenés et les autorités organisaurices, LNGV et le Verkehrsverbund Ostwestfalen-Lippe ont lancé un appel d'offres et: l'ensenble est repris par Eurobahn composé de la régie municipale des transports, l'Extertabahn ex de Euobahn filiales de deux partenaires Rhenus et Keolis. Le service commercial a débuté fin 2003. Cette ligne échappe à la NWl3 ex par conséquent au groupe CONNEX qui est en concurrence avec les groupes Rhenus et Keolis.

Même si les opérations précédentes jouent un rôle important dans leur territoire, le Teilnetz dénommé lans-Senne-Weser - ESW - occupe une place privilégiée. Ce réseau constitué par les lignes Münster-Bielefeldl'aderborn-Holzminden se développe sur 240 kilomètres et il s’inscrit pour sa plus grande partie dans le Land de Rhénanie du nord Wesphalie et pour quelques kilomètres dans celui de Basse-Saxe. Néanmoins ce Teilnetz est 


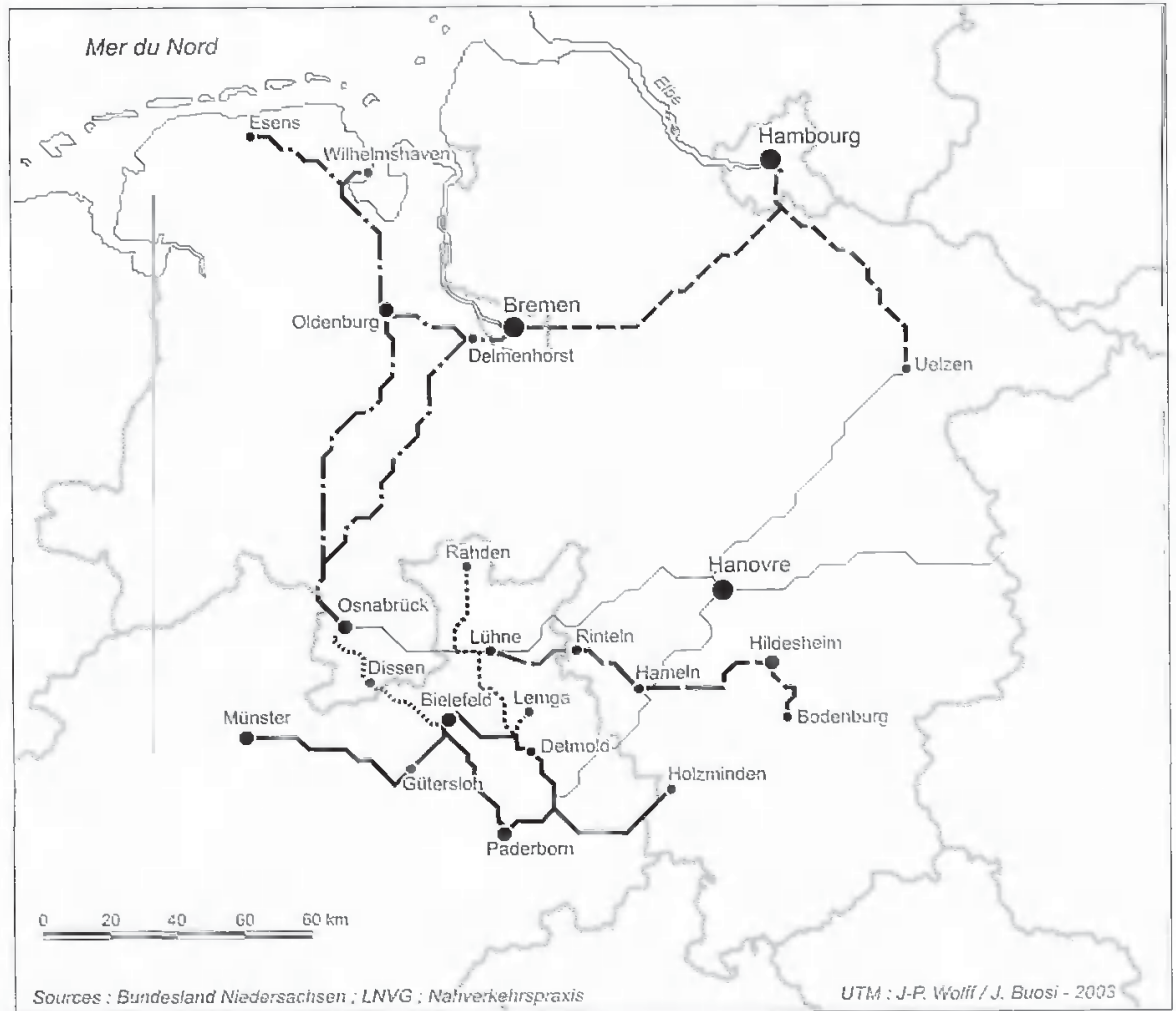

Soures : Sumdesland Nhadersthsen: LNVG ; Nahthehefrspraxis

- Principales lignes exploitées par la DB

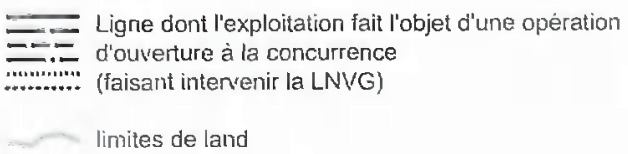

Fig. 2 Les relations ferroviaires ouvertes à la libéralisation en 2003.

The railway lines opened to the liberalization in 2003.

très proche de celui de la NWB partant d'Osnabrück. Les autorités organisatrices ont choisi un consortium associant la NWB ct la Teutohuger Wald Eiscubahn - TWE - qui est né fin 2003. A tuavers la constitution de ce réseau, une opportunité se dessine pour la NWB et done CONNEX de contrôler un ensemble ferroviaire de 720 kilomères, avec 7,5 millions de $\mathrm{km} / \mathrm{train} / \mathrm{an}$ et transportant près de 8 millions de passagers de part et d'autre d'Osnabrück. Les denx réseaux situés dans deux Länder différents dépendent de deux autorités organisatrices qui appliquent chacune dans leur Land leur tutelle sur la NWB. Done dans ce cas il y a création d'un mêne réseau technique, mais qui se décline en deux résenux d'exploitation ancrés non seulement dans les Länder en question mais de plus en plus 
dans des territoires plus petits er plus typés. Cette politique de la NWT3 correspond à sa volonté de devenir un acteur social et économique participant au développement local par sa politique de transport. Elle s'identifie à ces caractéristiques locales à travers des livrées du matériel faisant référence à un bassin de vie ou à une entité géographique à forte prégnance culturelle. Autou de Bielefeld, Munster et Osnabrück, trois agglonnérations séparées de 50 à 60 kilomètres les unes des autres et regroupant plus de 800000 habitants se développe un réseau de plus de 700 kilomètres dans le nordest de l'Allemagne et qui est centré autour d'Osnabrï̈ck, nême si début: 2004 il manque encore quelques kilomètres entre Osnabrück et Dissen pour son achèrement.

La première expérience réussie de la régionalisation et de la libéralisation conduite par la NWB dans le Land de Basse-Saxe a donné l'occasion à la LNGV de lancer d'autres operations identiques. Néanmoins si le succès a été au rendez-vous de cette première ouverture à la concurrence, il ne faut pas oublier de souligner la complexté des processus entre intervenants différents, aux logiques propres et souvent concurrentielles. Ce partenariat public-privé en répondant aux souhaits du Land avee plus de trains, plus de rapidité dans les trajets et l'introduction de I'horaire cadencé pour un méne coùt, rapprochent les aspirations sociales en matière de déplacement et les impératifs budgétaires.

L'introduction de la libéralisation dans ce cas correspond à une volonté des autorités du Land et des différentes villes concenées d'améliorer l'offre ferroviaire de proximité. Le choix d'une société privée à capitaux étrangers, même s'il pose quelques interrogations sur la non-réciprocité en France d'une telle politique pour l'heure interdice tant qu'une loi ne le permet pas, souligne les convergences entre des acteurs politiques fort éloignés les uns des atures et la demande sociale plus sensible à l'amélioration du service renouvelé qu'au maintien d'un service public trop éloigné des préoceupations des populations locales. I'efficacité de la société privée rejaillit sur les municipalités de gauche et l'image de service public dédouane CONNEX des exec̀s de la privatisation à l'anglaise. Les deux partenaires après avoir mis en place une structure de management du personnel qui fait cohabiter une direction à deux têtes pour gérer le réseau ont voulu ct su trouver une nouvelle respectabilité én offrant un service ferroviaire de grande qualité aux usagers potentiels et en se plaçant d'emblée dans une dénarche de commercialisation offensive et non en restant sur une rente de service public obsolète. La création des Netzteil obligeant l'opérateur retenu à prendre en charge une ligne peu fréquentée et à la tynamiser en offrant un service identique à celles du reste du réseau en temes de matériels, de cadencement, de correspondances est un facteur de développenent du uransport ferroviare dans des régions où jusqu'alors il reculait. Ajoutons qu'en remes de développement: territorial, cette mesure apporte un service amélioré dans le cas des espaces les moins favorisés socio-démographiquement mais en plus, elle s'inscrit dans une politique multimodale de transport. En 
effet, comme les princjpales villes via leurs services industriels gèrent les transports collectifs urbains et suburbains, elles peuvent peser dans l'articulation des complénentarités entre l'offece de transports collectits urbains et celle de la NW13 et ceci d'autant plus facilement qu'elles participent au capital de cette entreprise ferroviaire.

L'association de partenaires - privé et public - renforce les effets induits par la régionalisation qui sont de mener au plus près du terrain une politique qui réponde à l'attente potentielle des usagers et des acteurs politiques et économiques. La responsabilisation induite dans ee mariage de raison entraine une nouvelle dynamique et amplifie celle qui est produite par l'introduction de la régionalisation. Les réseaux institutionnels, politiques et techniques entre les deux partenaires de la NWB renforcent les syuergies communes et les logiques de fonctionnement des deux partenaires. La ville d'Osnabrück à travers les Stadtwerke s'implique considérablement en movens humains avec en particulicr les propositions du bureau d'études PLANOS contrôlé en partie par la commune, la mise à disposition de quelques agents des Stadwerke pour la nouvelle compagnie ferroviare et la nomination d'un codirecteur à la tête de la NWI3. Mais à còté de ces investissements en matière grise, Osnabrück a construit l'atelier de maintenance de la NWB sur ses propres emprises foncières et elle a racheté une ancienne gare inutilisée pour créer un point supplémentaire de desserte dans la ville. Cer investissement multiforme de la ville d'Osnabrück a joué un rôle non négligeable aux yeux de CONNEX en recherche de partenaires pour développer un nouveau réseau. Le savoir faire de la multinationale française n'a pu que séduire en matière de gestion de réseaux de transports ferroviaires de proximité des municipalités socialo-écologistes qui, malgré une implication déjà très forte à travers leur régie de transports urbains intégré aux Stadwerke, n'axaient qu"une expérience très limitée du transport ferroviaire. Osnabrück possède en effet un petit réseau en relation avec lactivité fret du port fluvial.

\section{Conclusion}

La régionalisation couplée à la libéralisation dans es contexte de partenariat à plusieurs entrées et encadrée par une convention précise et exigeante pour l'ensemble des partenaires montre que le transport ferroviaire de proximité ou régional peut devenir un facteur essentiel des politiques de déplacements et que de surcroît il s'inscrit également dans une politique de déreloppement territorial en intégrant les besoins des populations et des acteurs économiques. La réussite de cette politique dépend essentiellement de la bonne imbrication public/privée. Ajoutons qu'il répond à l'injonction de développement soutenable ou durable par son inscription dans une politique environnementale notamment marquée par le transfert vers le rail d'un nombre important de pendulaires, auparavant captif's de l'automobile, même lorsque les lignes ne sont pas électrifiées. Ce succès est une réponse aux défis posés par les 
déplacements à l'échelle locale et régionale et il va de soi qu'en Allemagne, les expériences menées et qui seront lancées permetront de renforeer les effets territoriaux en termes de cohérence et de cohésion de cette politique. Les enseignements de cette régionalisation/libéralisation questionnent les autorités organisatrices d'autres paýs européens. Le Royaume-Uni, chantre d'une certaine libéralisation introduit des correctifs à celle-ci avec la mise en place d'une autorité organisatrice chargée de réguler les dystonctionnements induits par la privatisation sauvage des années Thatcher et en particulier celle de l'infrastructure. D'autres États comme la France seront amenés, sous la pression du droit européen, à répondre à cette ouverture du secteur. ferroviaire et CONNEX qui fait ses armes à l'étranger pourait devenir un concurrent très sérieux de la SNCF sur les lignes non ou peu rentables selon les critères de la société nationale. Cet exemple dans ee contexte réglementaire et territorial particulier montre que l'immobilisme et le démantèlement d'un réseau ferroviaire de proximité peuvent être dépassés, comme le prowve l'expérience de la NordWestBalun en Basse-Saxe, grâce en partie ì la bonne coordination d'une multiplicité d'acteurs, témoignage d'une bonne "gouvernance» dans le domaine des transports ferroviaire.

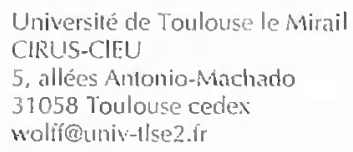

\section{Bibliographie}

Amslar Y. (1998), "Bilan de dix ans de déréglenzentation el de privatisation des transports urbains en Grande-Brelagne», Transports urbains, n 99 , p. 3-14.

Beaucire F, et Lebteton J. (2000), Transports publics et gouvennance urbaine, Milan, Toulouse, 127 p.

CERTU (1999), La desserte ferrée urbaine et périurbaines, tome 3: en Allemagne et en Suisse les SBahm, Lyon, 134 j.

Buddruss E. (1998), « Le trafic ferroviaire régional en Allemagne deux ans après la prise en charge par les Länder $x$, Transports urbains, n 99, p. 11-14.

Hannover Region (1997), Nahverkehsplan, Beiträge zur Regionalen Enwickhung, Hannover, 199 p.

Holz-Rau H.-C. (1996), "Est-il possible d'économiser les transports?", in La recherche sur la ville en Allemagne, Paris, CNRS, PIR-Villes, p. 111-126.

Kandler U. (2003), «10 Jahre DB AG», Eisenbahn Journal, Fürstenteldbruck, p. 6-11.

Kaufmanı V. (2000), Mobilité quotidienne el dynamiques urbaines, la question du rapport modal, Lausanne, Presses polytechniques et universitaires romandes, EPFL, $252 \mathrm{p}$.

Marconis R. (2000), "Les transports en France. Les enjeux territoriaux de la libéralisation el de la déréglementation $*$, Historiens ef Géographes, n 370, p. 299-319.

PLANOS (1997), Nahverkehrsplan rür die Stadt Osnabrück, Osnabrück, 255 p.

Séguret 5. (2003), La politique de libèralisation ierroviaire en Allemagne: I'exemple de la NordMestBahn en Basse-Saxe, ménoire de maîrise, Université d'Orléans, $126 \mathrm{p}$. 
Von Thünen J.-H. (1926, réédité 1990), Der isolierte Staat in Beziehung aứ Landwirtschaít und Naliönalökonomie, Scienta Verlag. Aalen, 678 p.

Wolff ).-P. (2002), "Les déplacements sous contraintes enviromementales", Pouvoirs locaux, $17^{\circ} 53$, p. $117-122$.

Ziegler B. (1999). "Le transport public allemand en question», La Vie du rail et des transports, n 105.

Ziegler B. (2000), "Allemagne: les régions maîtres du jeu \#, La Vie du rail et des transports, $n^{\circ} 125$. 